\title{
Three-dimensional analysis of accuracy of component positioning in total knee arthroplasty with patient specific and conventional instruments: A randomized controlled trial
}

\author{
Raf De Vloo ${ }^{\mathrm{a}, *}$, Pim Pellikaan ${ }^{\mathrm{b}}$, Aad Dhollander ${ }^{\mathrm{a}}$, Jos Vander Sloten ${ }^{\mathrm{b}}$ \\ a AZ Klina, Department of Orthopedic Surgery and Traumatology, Augustijnslei 100, 2930 Brasschaat, Belgium \\ ${ }^{\mathrm{b}}$ KU Leuven, Department of Mechanical Engineering, Biomechanics Section, Celestijnenlaan 300C, 3001 Leuven, Belgium
}

\section{A R T I C L E I N F O}

\section{Article history:}

Received 5 January 2017

Received in revised form 23 August 2017

Accepted 30 August 2017

Available online $\mathrm{xxxx}$

\section{Keywords:}

Total knee arthroplasty

Alignment

Patient specific guides

Patient specific instrumentation

3D analysis

Randomized controlled trial

\begin{abstract}
A B S T R A C T
Background: Component malalignment remains a major concern in total knee arthroplasty (TKA). Patient-specific guides (PSG) were developed to increase accuracy of bone resections and component placement, but available evidence is contradictory. We assessed the accuracy of 3D component placement in TKA with PSG compared to conventional surgery using virtual 3D bone models.

Methods: Fifty patients were randomly assigned to the PSG or conventional instrumentation group, 44 were finally analyzed. Preoperatively, MRI and CT scans were converted into virtual 3D models and a surgical plan was developed. Surgery was performed and changes in component sizing were recorded. Postoperative CT images were converted to 3D models and aligned to the planned, preoperative models and implant orientation. Differences between planned and postoperative implant orientations were calculated in 3D.

Results: PSG allowed significantly more accurate varus/valgus placement for the femoral component (PSG: $0.14 \pm 1.47$; control: $1.40 \pm 1.99 ; \mathrm{p}<0.05$ ), but more slope was introduced (PSG: $2.82 \pm 2.42$; control: $0.90 \pm 2.28 ; \mathrm{p}<0.05$ ). Less variability in positioning accuracy for femoral flexion angle and tibial rotation was found with PSG, indicating a result closer to the planned position, but no significant differences in positioning accuracy were found. PSG allowed more accurate prediction of the femoral (PSG: 100\%; control: 64\%) and tibial (PSG: 79\%; control 56\%) component size.

Conclusion: PSG led to adequate component positioning accuracy compared to the pre-operative plan. For the femoral component, the positioning was significantly closer to the planned position in the coronal plane, a similar trend was observed for the sagittal plane. But, for the tibial component, significantly more slope was introduced. A better prediction of component sizing was found with PSG compared to conventional surgery.
\end{abstract}

(c) 2017 Elsevier B.V. All rights reserved.

\section{Introduction}

The aim of total knee arthroplasty (TKA) is to relieve pain and to restore joint function on the longer term with a high patient satisfaction rate. Accurate positioning of the components leads to better stability of the joint, better loading conditions and

\footnotetext{
* Corresponding author.

E-mail addresses: raf.de.vloo@klina.be (R. De Vloo), Pim.Pellikaan@kuleuven.be (P. Pellikaan), Jos.vandersloten@kuleuven.be (J. Vander Sloten).
} 
therefore longevity of the implant [1,2]. These surgical and clinical parameters are associated with better short term clinical outcomes [3,4]. Poor alignment may lead to premature polyethylene wear, low functional scores and early failure [5]. Therefore, accurate bone cuts and final coronal, sagittal and axial alignment of the implant are essential. In clinical practice, the accuracy of component position is usually evaluated by the principle of $\pm 3^{\circ}$ deviation from neutral alignment. Although this principle is widely accepted, the clinical significance is not supported by scientific evidence and this alignment concept has consequently been questioned by some authors [6-8]. For conventional surgery, the incidence of component malalignment, independent of the surgical experience can be up to $30 \%$ [9].

Despite the widespread use of patient-specific guides (PSG) [14] there is still no clear scientific evidence on the validation of the guide's accuracy and superiority to conventional surgery or CAS [15]. The assessment of appropriate component position based on the rule of $\pm 3^{\circ}$ alignment is in the majority of the published studies made using plain radiographs [9-12,16,17]. This 2D (two dimensional) method however, has limitations in terms of accuracy and validity to evaluate a 3D (three dimensional) planning based on CT (computerised tomography) or MRI (magnetic resonance imaging) [18-20]. Furthermore, differences in use and definition of measured parameters to evaluate implant positioning make it difficult to correctly compare results of different studies [17]. A study of Boonen et al. [21] examined the accuracy of implant positioning with PSG by comparing the post-operative alignment to the preoperatively made digital planning, based on 3D CT-models. In this study the ranges of deviation from the pre-operative planning were compared to data available in the literature since no control group was present.

This prospective randomized controlled trial comparing MRI based PSG and conventional instrumentation was designed to address the following research questions. First, is there a difference in the accuracy of femoral or tibial component positioning as measured using CT 3D measurement techniques? Secondly, is there a difference in component sizing?

\section{Materials and methods}

\subsection{Study design}

From April 2014 to November 2014, 52 patients scheduled for TKA were included in the study. Inclusion and exclusion criteria according to the general criteria for TKA with the PSG technique were followed (Table 1). Surgery was canceled for two patients due to cardiac conditions and psychological problems respectively. In total 50 patients scheduled for primary TKA surgery were included (Figure 1). The study was approved by the local ethical committee (EC025/200/013) and all included patients signed an informed consent. Four surgeries were planned per day. Randomizations were done the day of surgery by an independent person who tossed a coin to define the two patients that would be operated using conventional instrumentation. This trial complies with the CONSORT statement.

\subsection{Pre-operative measurements}

Preoperatively, MRI scans were obtained for all patients according to the standard Signature scanning protocols and sent to the manufacturing company (Materialise NV, Leuven, Belgium) for further processing. Standard procedures were followed according to the Signature MRI total knee system (Biomet, Inc., Warsaw, Indiana, United States). MRI images were converted to virtual 3D models (Mimics, Materialise NV, Leuven, Belgium), anatomical landmarks were selected for definition of the coordinate system and a virtual planning was developed and proposed to the surgeon (Biomet Signature Planner, Materialize NV, Leuven, Belgium).

Table 1

Inclusion and exclusion criteria.

Inclusion criteria for this study

- Painful and disabled knee joint resulting from osteoarthritis.

- One or more compartments are involved, as assessed by X-ray.

- High need to obtain pain relief and improve function.

Exclusion criteria for this study

- Active infection in the knee

- Body Mass Index (BMI) > 38

- Age $<50$ years old.

- General infection

- Distant foci of infections which may spread to the implant site

- Failure of previous joint replacement

- Pregnancy

- Previous major knee surgery, except for arthroscopic meniscectomy

- High sensitivity to radiation

- Metal within $10 \mathrm{~cm}$ of knee joint

- Presence of pacemaker, neuro-stimulator or cerebral shunt

- Claustrophobia 


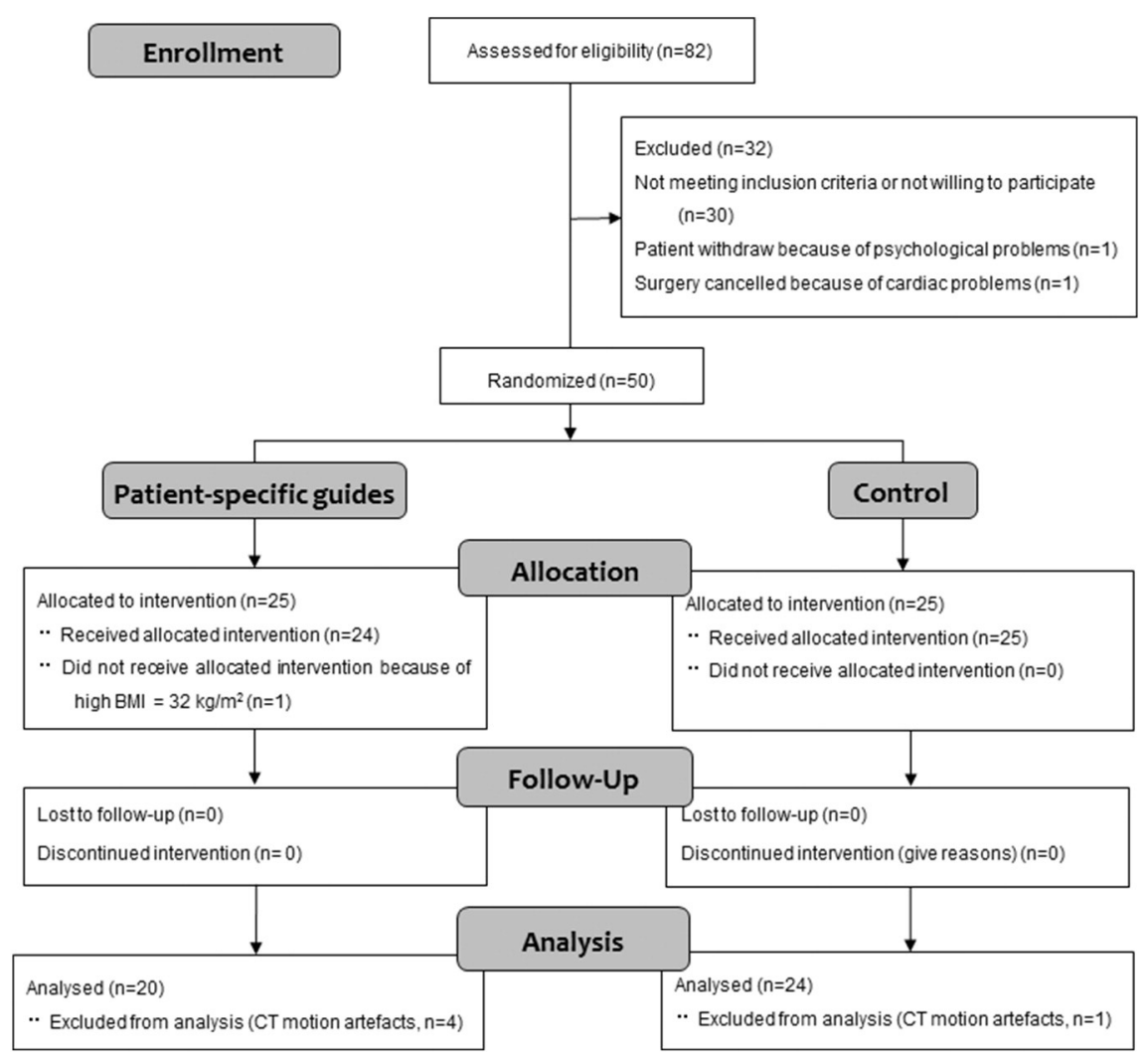

Figure 1. Patient flow diagram.

The Signature software allows modifications of implant size, resection level and component orientation in the coronal, sagittal and axial planes. A default planning was proposed to the surgeon who made changes where needed and gave his final approval.

Additionally, each patient had a pre-operative full leg CT-scan. CT-images were segmented (RMS (root mean square) error of $0.55 \mathrm{~mm}$ [22]) using the Mimics Innovation Suite (Materialise NV, Leuven, Belgium). The CT-scans were not used for surgical planning, but were required additional measurements for the post-processing of the data, to be able to link the post-operative CT-based model more reliable to the pre-operative MRI-based model for the accuracy assessment of component positioning.

\subsection{Operative technique}

The surgery was performed at a single site by a single surgeon with experience since April 2011 with the Signature patient-specific guides for the Vanguard Knee System (Biomet, Inc., Warsaw, Indiana, United States). Patients in both cohorts received either a posterior-stabilized (PS) or a cruciate retained (CR) Vanguard knee prosthesis. The choice to use a particular implant type was made perioperatively depending on the severity of arthritis and the state of the cruciate ligaments. All TKAs were performed using a midvastus approach without patella eversion and without the use of a tourniquet.

For the control group, the Signature surgical plan (anatomic femoral valgus angle, resection levels and sizes) was not consulted prior to or during the surgery. For the conventional group, the distal femoral resection was made using an intramedullary rod with a valgus angle of five degrees [22,23] and a resection level of nine millimeters from the medial condyle. A pre-operative full leg radiograph was not done and a fixed femoral valgus angle of five degrees was used for alignment. The proximal tibial cut was made using extramedullary instrumentation. The resection level depended on the worn anatomical structures and a visual estimation of the natural slope from the medial tibia plateau. The medial third of the tibial tuberosity was used to determine the rotation of the tibial component. The femoral rotation was determined by flexion spacer blocks with the knee at $90^{\circ}$ flexion and the patella in place. Reconstruction of the posterior condylar offset while conserving the level of the joint line was the target in both groups. Resection of all osteophytes and full posterior capsular release were performed if necessary to regain full extension. In case of a tight flexion and extension gap extra resection of the proximal tibia was performed. Extra resection was only performed in the coronal plane on the femur or tibia and never in the sagittal or axial plane. Ligament release was done if necessary to balance the ligaments to obtain a rectangular joint space. For the Signature PSG group, the pins and associated cut blocks were placed according to the pre-operatively planned position and the surgical plan was referenced intraoperatively. The implanted component sizes and intraoperative modifications of the approved PSG planning were recorded. 


\subsection{Post-operative measurements}

Postoperatively (average 46 days, range 31-81 days), a full leg CT scan was obtained for all patients using the same scanning protocol as preoperatively to calculate the differences between the preoperative plan and postoperative measurements. Three dimensional virtual bone models were derived from the Signature planning and postoperative CT images using the Mimics Innovation Suite (Materialise NV, Leuven, Belgium). First, CAD (computer aided design) models of the implanted components were superposed on their segmented postoperative counterparts. Second, the postoperative CT bone models were registered on the preoperative CT bone models using an iterative closest point (ICP) algorithm. Similarly, the pre-operative CT bone models were registered on the Signature planning models using the same method and manually transformed to the same coordinate system as used preoperatively to perform the planning. The same transformation was applied to the CAD models of the components. Thirdly, we measured the orientation of the CAD models in the post-operative 3D model and calculate the difference with the planned orientation in the coronal, sagittal and axial planes (Figure 2). Femoral varus angle deviation was defined as the angle between the intersection lines of the pre- and postoperative distal cut planes with the femoral coronal plane. Positive values indicate a varus position, negative values a valgus position. Femoral flexion angle deviation was defined as the angle between the intersection lines of the post-operative distal cut planes with the femoral sagittal plane. Positive values indicate flexion, negative values extension. Femoral rotation was defined as the angle between the intersection lines of the pre- and postoperative cut planes with the femoral axial plane. Positive values indicate an external rotation, negative values an internal rotation. Tibial varus angle deviation was defined as the angle between the intersection lines of the pre- and postoperative proximal cut planes with the tibial coronal plan. Positive values indicate a varus position, negative values a valgus position. Tibial slope angle deviation was defined as the angle between the intersection lines of the pre- and postoperative proximal cut planes with the tibial sagittal plane. Positive values indicate an upslope, negative values indicate a downslope. Tibial rotation was defined as the angle between the tibial anterior-posterior line projection on the tibial axial plane and intersection line of the post-operative tibial implant symmetry plane with the tibial axial plane. Positive values indicate an external rotation, negative values an internal rotation.

\subsection{Statistical analysis}

A power analysis (Minitab Inc., State College, PA, USA), indicated that 26 patients per group would be required for this trial to detect a component alignment difference of one degree measured on the 3D model with a significance level of 0.05 and $80 \%$ power. An unpaired sample Student's t-test was used to establish the differences in component positioning, expressed the difference between pre-op plan and post-op position, between the PSG group and the control group based on individualized 3D models. Level of significance was set to $\mathrm{p}<0.05$ (Minitab Inc., State College, PA, USA).

\section{Results}

For two included patients the surgery was canceled because of psychological problems and cardiac problems. One subject in the PSG group with a Body Mass Index (BMI) of $38 \mathrm{~kg} / \mathrm{m}^{2}$ was excluded because the surgical technique was intraoperatively changed from PSG to conventional. Despite adequate surgical exposure, very hard and stiff subcutaneous fatty tissue did not allow for stable positioning of the PSG guide on the proximal tibia with adequate visualization of the position and slope. During the post-operative analysis, four subjects in the PSG group and one in the control group had to be excluded because of motion artifacts during preoperative or postoperative CT-imaging, which made further processing impossible (Figure 1). Since the total resection measurements with the Signature planning cannot account for soft tissue laxity, fine-tuning of the resection level is

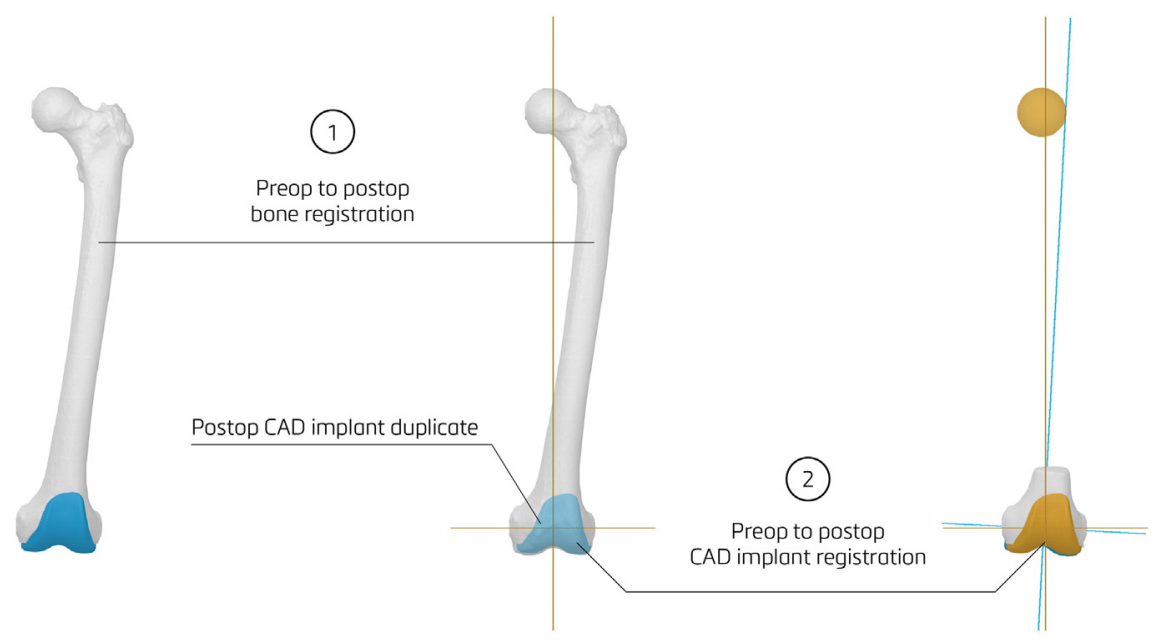

Figure 2. Illustration of the workflow for creation of the 3D virtual models. 
necessary to achieve optimal results and can only be done during the surgery. The final implant position can only be decided intraoperatively for an optimal balancing in flexion and extension. For the 25 patients in the PSG group, 11 extra femoral resections (nine times two millimeters and two times three millimeters) and three extra tibial resections (three times two millimeters) were made to equalize the flexion and extension gap and to adjust for ligament tension. The overall component and limb alignment was not changed by these extra resections who were only performed in the coronal plane (distal femur or proximal tibia) and never in the sagittal or axial plane. In both groups ligament release was done if necessary to balance the ligaments to obtain a rectangular joint space. Ligament release was performed in four of the 25 patients in the PSG group (two times medial and two times lateral) and in two of the 25 patients in the control group (one time medial and one time lateral).

Both groups showed similar demographics: The PSG group consisted of 14 females and 11 males. Mean BMI was $29.24 \mathrm{~kg} / \mathrm{m}^{2}$ (SD (standard deviation) 3.57) and mean age 72.72 (SD 8.89). The control group consisted of 15 females and 10 males. Mean BMI was 29.92 (SD 4.27), mean age 72.28 (SD 7.99). In both groups, the primary diagnosis was osteoarthritis for all patients.

Table 2 presents the deviations from the pre-operatively planned component positioning to the postoperative positioning, compared between both groups. The PSG group showed significant more accurate varus/valgus positioning of the femoral component compared to the control group $(\mathrm{p}<0.05)$ and all femoral components were placed within the range of $-3.3^{\circ}$ to $3.0^{\circ}$ compared to a range of $-2.9^{\circ}$ to $6.4^{\circ}$ for the control group (Figure 3a). No significant differences were found for femoral flexion/extension, but a smaller range of deviation from the target position was present (PSG: $-3.0^{\circ}$ to $6.4^{\circ}$; control: $-5.1^{\circ}$ to $7.5^{\circ}$ ) together with a smaller SD, indicative for a trend towards a resulting positioning closer to the target or planned positioning with PSG (Figure $3 \mathrm{~b}$ ). For femoral rotation, no significant difference in component positioning accuracy was found (Table 2), but the range of position deviations was larger in the PSG group (PSG: $-3.5^{\circ}$ to $6.2^{\circ}$; control: $-2.5^{\circ}$ to $4.7^{\circ}$; Figure $3 \mathrm{c}$ ). The tibial component positioning showed a smaller deviation range for varus/valgus angle in the PSG group (PSG: $-1.8^{\circ}$ to $3.6^{\circ}$; control: $-3.6^{\circ}$ to $3.3^{\circ}$; Figure $4 \mathrm{a}$ ). Component positioning was the least accurate for tibial rotation for both the PSG and conventional groups, in which the highest deviations from the target position were found (PSG: $-10.6^{\circ}$ to $11.5^{\circ}$; control: $-9.4^{\circ}$ to $21.4^{\circ}$; Figure $4 \mathrm{c}$ ). With PSG, significantly more tibial slope (1.92 ${ }^{\circ}$ on average, $\mathrm{p}<0.05$ ) with reference to the planned position was introduced. Ranges of deviation from the planned position were $-3.3^{\circ}$ to $7.1^{\circ}$ for PSG and $-3.0^{\circ}$ to $5.3^{\circ}$ for the control group (Figure $4 \mathrm{~b}$ ). Fourteen patients in both the study and control groups received a CR prosthesis, six patients in the study group and 10 patients in the control group received a PS prosthesis. When dividing groups by prosthesis type, a statistical significant difference in average accuracy was only observed in the group receiving a CR prosthesis with respect to femoral rotation (PSG: $0.5^{\circ}$; control: $2.0^{\circ} ; \mathrm{p}<0.05$ ) and tibial slope (PSG: $3.0^{\circ}$; control: $0.7^{\circ} ; \mathrm{p}<0.05$ ). No significant differences between prosthesis types were observed in the control and study groups, respectively.

The femoral component size was $100 \%$ correctly planned in the PSG group, while in the control group the size had to be changed in $36 \%$ of the cases. The tibial component size was perioperatively changed to one size smaller than the planned size for $21 \%$ of the cases in the PSG group, while in the control group the planned size had to be up- or down-sized in $44 \%$ of the cases. In the control group, the surgeon was blinded to the planned sizes.

\section{Discussion}

We compared the accuracy of component positioning in TKA with PSG to conventional surgery measured in 3D on virtual bone models in a randomized clinical trial. Our results point to component positioning closer to the planned positioning with PSG for femoral varus/valgus and flexion/extension and for tibial rotation for the tibial component, but the results were only statistically significant for femoral varus/valgus. Larger sample sizes will be needed to confirm the observed trends. However, higher accuracy with PSG was not confirmed for femoral rotation and tibial slope. Previous studies on the accuracy of component positioning with PSG showed contradictory results. Several systematic reviews and meta-analyses were conducted to formulate general conclusions on the use of PSG compared to conventional techniques [9,17,24-29]. Superior accuracy of alignment with PSG could not be demonstrated, but the authors mentioned low methodological quality, small patient cohorts and low statistical power, lack of homogeneity and a high risk of bias in the analyzed studies as a strong limitation to draw final conclusions. To date, studies with sufficient statistical power and reporting level 1 evidence on the use of PSG are scarce. The majority of the studies that analyze accuracy of component positioning in TKA use measurements on plain radiographs, which is another important limitation. Hirschmann et al. [19] compared conventional radiographs and 3D-CT models for the assessment of component positioning in TKA.

Table 2

Deviations from planned alignment compared for the PSG and conventional group.

\begin{tabular}{|c|c|c|c|c|c|c|c|}
\hline & \multicolumn{3}{|c|}{ Study group (PSG) } & \multicolumn{3}{|c|}{ Control group } & \multirow[b]{2}{*}{$\mathrm{p}$} \\
\hline & $\mathrm{N}$ & Mean & SD & $\mathrm{N}$ & Mean & SD & \\
\hline Femoral varus angle deviation $\left({ }^{\circ}\right)$ & 20 & 0.14 & 1.47 & 24 & 1.40 & 1.99 & $0.021^{*}$ \\
\hline Femoral flexion angle deviation $\left({ }^{\circ}\right)$ & 20 & 2.04 & 2.37 & 24 & 2.23 & 3.32 & 0.833 \\
\hline Femoral rotation $\left({ }^{\circ}\right)$ & 20 & 0.85 & 2.27 & 24 & 1.65 & 1.97 & 0.228 \\
\hline Tibial varus angle deviation $\left({ }^{\circ}\right)$ & 20 & 0.58 & 1.66 & 24 & -0.34 & 1.81 & 0.086 \\
\hline Tibial slope deviation $\left({ }^{\circ}\right)$ & 20 & 2.82 & 2.42 & 24 & 0.90 & 2.28 & $0.011^{*}$ \\
\hline Tibial rotation $\left({ }^{\circ}\right)$ & 20 & -1.42 & 6.28 & 24 & 1.61 & 9.00 & 0.198 \\
\hline
\end{tabular}

Negative values represent valgus in the coronal plane, extension or down slope in the sagittal plane, and internal rotation in the axial plane.

* Significant at $\mathrm{p}<0.05$. 
a

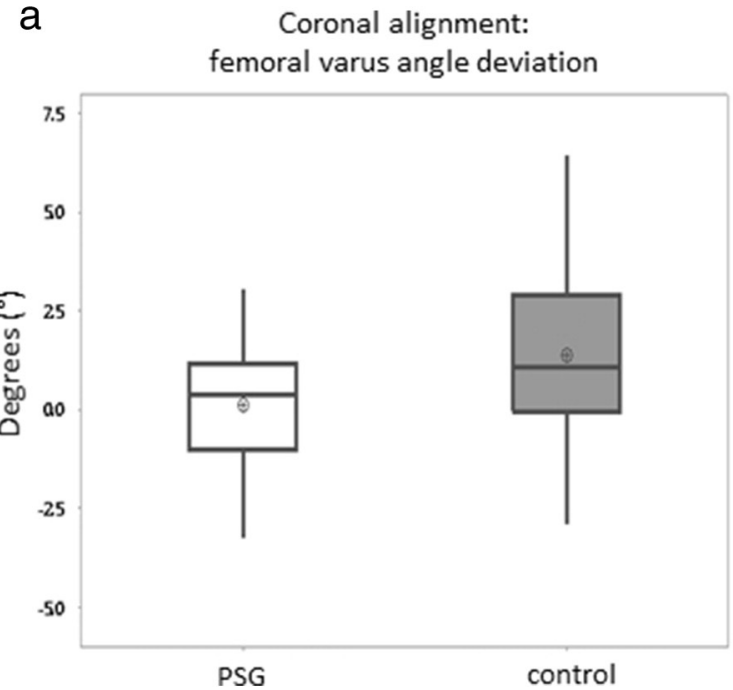

b

Sagittal alignment:

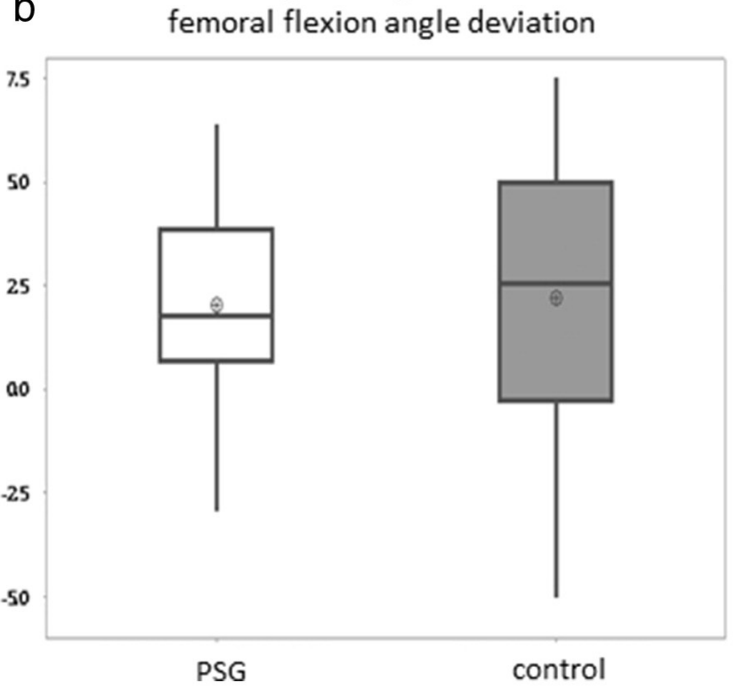

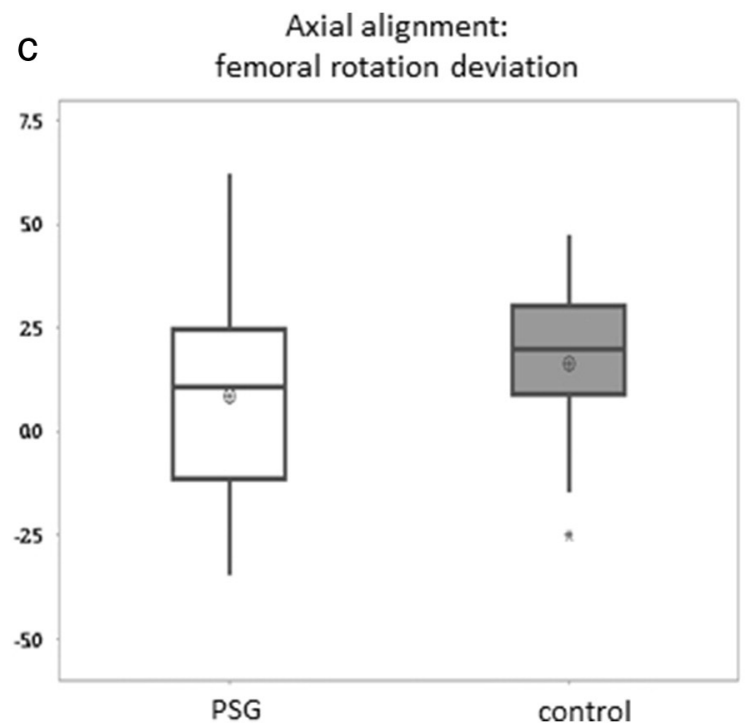

Figure 3. a: Deviation from planned alignment for the femoral component in the coronal plane, for the patient-specific guide (PSG) group and control group. b: Deviation from planned alignment for the femoral component in the sagittal plane, for the patient-specific guide (PSG) group and control group. c: Deviation from planned alignment for the femoral component in the transverse plane, for the patient-specific guide (PSG) group and control group.

Their results clearly show high inter- and intra-observer variability of plain radiographs indicating low clinical utility and reproducibility, whereas inter- and intra-observer variability on 3D-CT models was significantly better resulting in highly reliable measurements while using this method. Similarly, Holme et al. [20] found poor accuracy for plain radiographs compared to 3D-CT models as shown by a disagreement for all three parameters of tibial component orientation when comparing plane radiographs with 3D-CT models. On the other hand, Jonkergouw et al. [30] described a CT based 3D measurement method for evaluation of implant positioning accuracy in TKA, comparing post-operative implant position to the corresponding pre-operative plan using $3 \mathrm{D}$ virtual models. They showed measurement errors less than $1.0^{\circ}$ or $0.5 \mathrm{~mm}$. It is therefore likely that published studies using plain radiographs to assess component placement accuracy are limited by measurement error and the use of 3D-CT analysis is the only way to report with confidence on implant positioning accuracy [18]. However, to date little evidence based on 3D measurement methods can be found in the literature. In a prospective cohort study Boonen et al. [21] used the same method as in our study and concluded that the preoperative plane was adequately reproduced within a range of $\pm 3^{\circ}$ in all planes, except for tibial component rotation where the deviation was on average $3.2^{\circ}$. Several studies using measurement on CT slices such as a modified Perth protocol [31] have reported superior alignment accuracy with PSG compared to conventional instrumentation [32-37]. In two separate studies Heyse and Tibesku [38,39] analyzed rotational alignment with MRI and also observed the superior outcome of PSG compared to conventional technique in terms of outliers. Only Woolson et al. [40] reported no significant improvement in alignment with PSG and a higher prevalence of malalignment for tibial slope. 


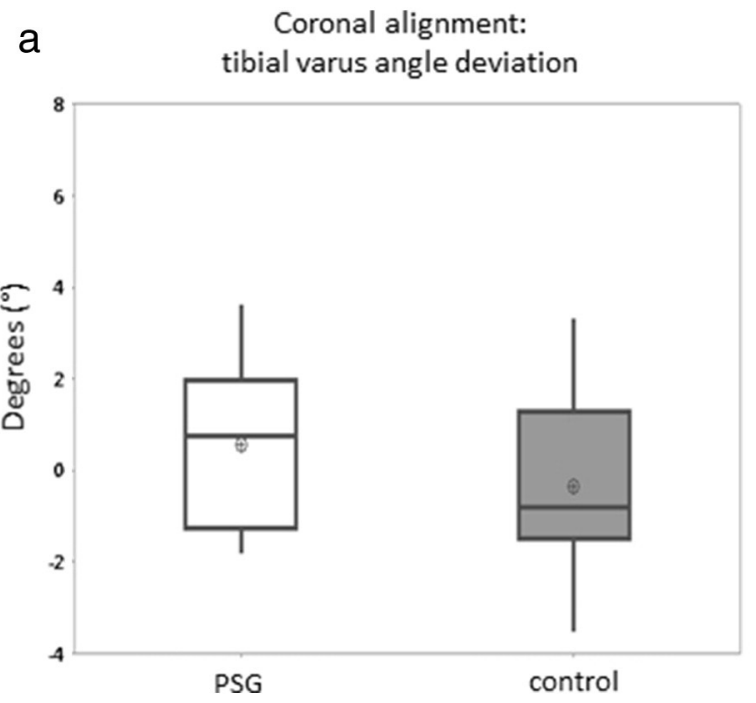

b

Sagittal alignment: tibial slope deviation

C

Axial alignment: tibial rotation deviation

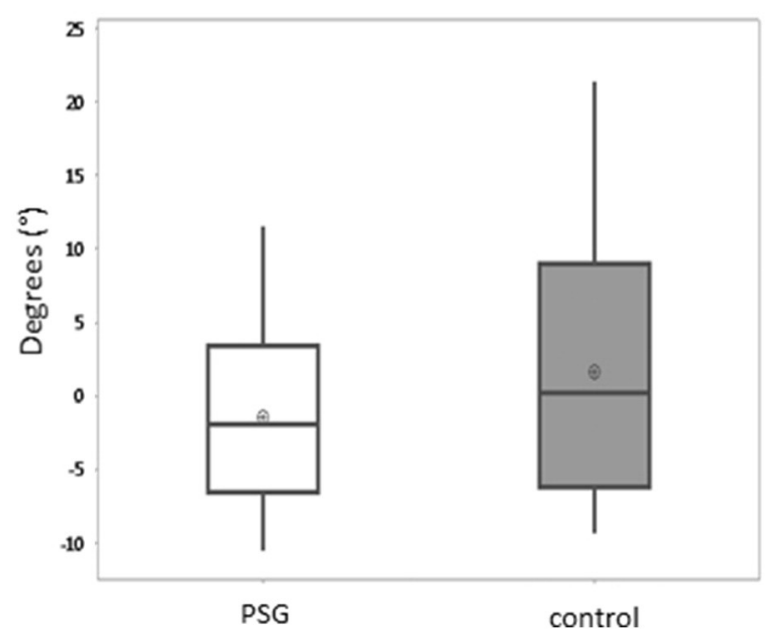

Figure 4. a: Deviation from planned alignment for the tibial component in the coronal plane, for the patient-specific guide (PSG) group and control group. b: Deviation from planned alignment for the tibial component in the sagittal plane, for the patient-specific guide (PSG) group and control group. c: Deviation from planned alignment for the tibial component in the transverse plane, for the patient-specific guide (PSG) group and control group.

Overall, the available evidence in literature shows that PSG demonstrate significant and consistent advantages in terms of alignment over conventional instrumentation in TKA when analyzed with 3D imaging methods. Our results confirmed this trend with reference to a control group, except for femoral rotation and tibial slope. Similarly to the study of Woolson et al. [40], we found less accurate tibial slope in the PSG group compared to controls. Based on our experience, it would be helpful to design a finger pit on the tibial guide (MRI-based), similar to the tibial CT-based guide, to enhance a good grip that would facilitate the positioning of the guide on the tibia plateau, which may improve the accuracy for slope and rotation.

Besides improvements in component placement accuracy, the use of surgical planning showed a clear advantage for predicting component sizing which was $100 \%$ correct for the femur and $79 \%$ for the tibia. In the conventional group, the preoperative planning was blinded to the surgeon. Changes in size were often needed which is unfavorable for in-hospital logistic and economic reasons.

Correct alignment in TKA is still a point of discussion and the concept of neutral alignment is questionable [6,41]. The surgical planning tool for TKA allows individualization of the pre-operative plan to the patient's anatomy and fine tuning of the default plan with clear 3D visualization of the modifications and the effect on limb alignment, component size, bony resections etc. The thickness of the combined tibial and femoral resections in flexion and extension can give an indication on perioperative ligament tension and can be taken into consideration to equalize the flexion and extension gap on the medial and on the lateral side. The surgeon has the possibility to analyze in detail whether a mechanical, kinematic or anatomical alignment would be beneficial or whether a neutral alignment or restoration of constitutional varus or valgus might be more appropriate, depending on each 
individual case. In the literature, correct alignment is often discussed based on outlier rates defined as a deviation of $\pm 3^{\circ}$ from a neutral position despite the absence of evidence to support the guideline [6-8]. Moreover, most studies have small sample sizes which is problematic for correctly reporting on outlier rates. An analysis of deviation from a planned position may therefore enhance the insights on component placement accuracy in TKA.

In the present study, extra resections on tibia and femur were needed to adapt for ligament tension during surgery. Since the planning tool cannot adapt for elastic properties of soft tissue, this fine-tuning is necessary to achieve optimal results and can only be done intra-operatively by the surgeon. The extra resection levels made were therefore not related to an error in guide design.

A limitation of the present study is the use of an extra CT scan which includes an extra radiation dose for the patient. However, the use of this additional scan was necessary because it significantly increases the accuracy of the registration of the preoperative MRI to the postoperative CT scan by adding an intermediate step to the registration process [21]. First, the postoperative CT scan is accurately registered to the preoperative CT scan by using the same scan protocol. Afterwards, the preoperative CT scan is registered to the preoperative MRI scan. In both scans the articulating ends of the femur and tibia are clearly visible. Consequently, registration can be done much more accurately than if the postoperative CT scan was used for immediate registration on the preoperative MRI, because the former contains the implant and resected bones. It was however a major concern to limit the radiation exposure for the patients. The scan protocol was tested for adequate parameter settings that generate images with sufficient accuracy and resolution for performing the measurements but at the lowest possible radiation dose. The total radiation exposure for the patients was $11.38 \mathrm{mSv}$. Based on the values published by the Radiological Society of North America and the American College of Radiology [41], this dose is comparable with one CT-pelvis or one CT-thorax. Furthermore, the lack of an immediate quality control of medical imaging is another major limitation of this study. Motion artifacts were the cause for five dropouts. These patients were not recalled for an extra CT to limit radiation exposure. Consequently, the sample size as calculated by the power analysis could not be reached and hinders the statistical analysis of our results [42].

In conclusion, PSG lead to better prediction of component sizing and to adequate component positioning accuracy compared to the pre-operative plan. Femoral varus/valgus positioning was significantly closer to the planned position, a similar non-significant trend was observed for flexion/extension. However, significantly more tibial slope was introduced. Additional studies on component accuracy placement evaluated with 3D imaging tools of PSG on larger patient cohorts are needed to demonstrate the added value of this technique.

\section{Acknowledgment}

The authors acknowledge Karim Chellaoui and Gerlinde Lenaerts (employees of Materialise, NV) for their support in the set-up of the study and manuscript writing.

\section{References}

[1] Gromov K, Korchi M, Thomsen MG, Husted H, Troelsen A. What is the optimal alignment of the tibial and femoral components in knee arthroplasty? Acta Orthop 2014;85:480-7. https://doi.org/10.3109/17453674.2014.940573.

[2] Ritter MA, Davis KE, Meding JB, Pierson JL, Berend ME, Malinzak RA. The effect of alignment and BMI on failure of total knee replacement. J Bone Joint Surg 2011; 93:1588-96. https://doi.org/10.2106/JBJS.J.00772.

[3] Choong PF, Dowsey MM, Stoney JD. Does accurate anatomical alignment result in better function and quality of life? Comparing conventional and computerassisted total knee arthroplasty. J Arthroplasty 2009;24:560-9. https://doi.org/10.1016/j.arth.2008.02.018.

[4] Yaffe M, Luo M, Goyal N, Chan P, Patel A, Cayo M, et al. Clinical, functional, and radiographic outcomes following total knee arthroplasty with patient-specific instrumentation, computer-assisted surgery, and manual instrumentation: a short-term follow-up study. Int J Comput Assist Radiol Surg 2014;9:837-44. https://doi.org/10.1007/s11548-013-0968-6.

[5] Michos J, Karachalios T. Long term clinical outcome of total knee arthroplasty. The effect of limp alignment, implant placement and stability as controlled by surgical technique. In: Karachalios T, editor. Total knee arthroplasty. London: Springer; 2015. p. 85-100.

[6] Bellemans J. Neutral mechanical alignment: a requirement for successful TKA: opposes. Orthopedics 2011;34:e507-09. https://doi.org/10.3928/0147744720110714-41.

[7] Bonner T], Eardley WGP, Patterson P, Gregg PJ. The effect of post-operative mechanical axis alignment on the survival of primary total knee replacements after a follow-up of 15 years. J Bone Joint Surg Br 2011;93-B:1217-22. https://doi.org/10.1302/0301-620X.93B9.26573.

[8] Parratte S, Pagnano MW, Trousdale RT, Berry DJ. Effect of postoperative mechanical axis alignment on the fifteen-year survival of modern, cemented total knee replacements. J Bone Joint Surg 2010;92:2143-9. https://doi.org/10.2106/JBJS.I.01398.

[9] Thienpont E, Schwab PE, Fennema P. A systematic review and meta-analysis of patient-specific instrumentation for improving alignment of the components in total knee replacement. Bone Joint J 2014;96-B:1052-61. https://doi.org/10.1302/0301-620X.96B8.33747.

[10] Hetaimish BM, Khan MM, Simunovic N, Al-Harbi HH, Bhandari M, Zalzal PK. Meta-analysis of navigation vs conventional total knee arthroplasty. J Arthroplasty 2012;27:1177-82. https://doi.org/10.1016/j.arth.2011.12.028.

[11] Mason JB, Fehring TK, Estok R, Banel D, Fahrbach K. Meta-analysis of alignment outcomes in computer-assisted total knee arthroplasty surgery. J Arthroplasty 2007;22:1097-106. https://doi.org/10.1016/j.arth.2007.08.001.

[12] Camarda L, D'Arienzo A, Morello S, Peri G, Valentino B, D'Arienzo M. Patient-specific instrumentation for total knee arthroplasty: a literature review. Musculoskelet Surg 2014;1:11-8. https://doi.org/10.1007/s12306-014-0339-7.

[14] Thienpont E, Bellemans J, Delport H, Van Overschelde P, Stuyts B, Brabants K, et al. Patient-specific instruments: industry's innovation with a surgeon's interest. Knee Surg Sports Traumatol Arthrosc 2013;21:2227-33. https://doi.org/10.1007/s00167-013-2626-5.

[15] Thienpont E, Fennema P, Price A. Can technology improve alignment during knee arthroplasty. Knee 2013;20:S21-8. https://doi.org/10.1016/S09680160(13)70005-X.

[16] Mannan A, Smith TO, Sagar C, London NJ, Molitor PJA. No demonstrable benefit for coronal alignment outcomes in PSI knee arthroplasty: a systematic review and meta-analysis. Orthop Traumatol Surg Res 2015;101:461-8. https://doi.org/10.1016/j.otsr.2014.12.018.

[17] Cavaignac E, Pailhé R, Laumond G, Murgier J, Reina N, Laffosse JM, et al. Evaluation of the accuracy of patient-specific cutting blocks for total knee arthroplasty: a meta-analysis. Int Orthop 2014;39:1541-52. https://doi.org/10.1007/s00264-014-2549-x.

[18] Delport HP, Vander Sloten J. Evaluation of patient specific instruments. To measure is to know! J Arthroplasty 2014;30:720-1. https://doi.org/10.1016/j.arth. 2014.11.026. 
[19] Hirschmann MT, Konala P, Amsler F, Iranpour F, Friederich NF, Cobb JP. The position and orientation of total knee replacement components: a comparison of conventional radiographs, transverse 2D-CT slices and 3D-CT reconstruction. J Bone Joint Surg Br 2011;93:629-33. https://doi.org/10.1302/0301-620X.93B5. 25893.

[20] Holme TJ, Henckel J, Cobb J, Hart AJ. Quantification of the difference between 3D CT and plain radiograph for measurement of the position of medial unicompartmental knee replacements. Knee 2011;18:300-5. https://doi.org/10.1016/j.knee.2010.07.010.

[21] Boonen B, Schotanus MGM, Kerens B, Hulsmans F-J, Tuinebreijer WE, Kort NP. Patient-specific positioning guides for total knee arthroplasty: no significant difference between final component alignment and pre-operative digital plan except for tibial rotation. Knee Surg Sports Traumatol Arthrosc 2015. https:// doi.org/10.1007/s00167-015-3661-1.

[22] Deakin AH, Basanagoudar PL, Nunag P, Johnston AT, Sarungi M. Natural distribution of the femoral mechanical-anatomical angle in an osteoarthritic population and its relevance to total knee arthroplasty. Knee 2012;19:120-3. https://doi.org/10.1016/j.knee.2011.02.001.

[23] Kharwadkar N, Kent RE, Sharara KH, Naique S. 5 degrees to 6 degrees of distal femoral cut for uncomplicated primary total knee arthroplasty: is it safe? Knee 2006;13:57-60. https://doi.org/10.1016/j.knee.2005.07.001.

[24] Jiang J, Kang X, Lin Q, Teng Y, An L, Ma J, et al. Accuracy of patient-specific instrumentation compared with conventional instrumentation in total knee arthroplasty. Orthopedics 2015;38:e305.

[25] Voleti PB, Hamula MJ, Baldwin KD, Lee G-C. Current data do not support routine use of patient-specific instrumentation in total knee arthroplasty. J Arthroplasty 2014;29:1709-12. https://doi.org/10.1016/j.arth.2014.01.039.

[26] Fu H, Wang J, Zhou S, Cheng T, Zhang W, Wang Q, et al. No difference in mechanical alignment and femoral component placement between patient-specific instrumentation and conventional instrumentation in TKA. Knee Surg Sports Traumatol Arthrosc Off J ESSKA 2015;23:3288-95. https://doi.org/10.1007/ s00167-014-3115-1.

[27] Shen C, Tang Z-H, Hu J-Z, Zou G-Y, Xiao R-C, Yan D-X. Patient-specific instrumentation does not improve accuracy in total knee arthroplasty. Orthopedics 2015; 38:e178-88. https://doi.org/10.3928/01477447-20150305-54.

[28] Russell R, Brown T, Huo M, Jones R. Patient-specific instrumentation does not improve alignment in total knee arthroplasty. J Knee Surg 2014;27:501-4. https:// doi.org/10.1055/s-0034-1368143.

[29] Sassoon A, Nam D, Nunley R, Barrack R. Systematic review of patient-specific instrumentation in total knee arthroplasty: new but not improved. Clin Orthop 2015;473:151-8. https://doi.org/10.1007/s11999-014-3804-6.

[30] Jonkergouw F, Allé F, Chellaoui K, Vander Sloten J, Vangeneugden D. Three-dimensional measurement technique to assess implant position and orientation after total knee arthroplasty. Med Eng Phys 2016. https://doi.org/10.1016/j.medengphy.2016.09.006.

[31] Chauhan SK, Clark GW, Lloyd S, Scott RG, Breidahl W, Sikorski JM. Computer-assisted total knee replacement. A controlled cadaver study using a multi-parameter quantitative CT assessment of alignment (the Perth Protocol). J Bone Joint Surg Br 2004;86-B:818-23. https://doi.org/10.1302/0301-620X.86B6.15456.

[32] Ferrara F, Cipriani A, Magarelli N, Rapisarda S, De Santis V, Burrofato A, et al. Implant positioning in TKA: comparison between conventional and patient-specific instrumentation. Orthopedics 2015;38:e271-80. https://doi.org/10.3928/01477447-20150402-54.

[33] Chotanaphuti T, Wangwittayakul V, Khuangsirikul S, Foojareonyos T. The accuracy of component alignment in custom cutting blocks compared with conventional total knee arthroplasty instrumentation: prospective control trial. Knee 2014;21:185-8. https://doi.org/10.1016/j.knee.2013.08.003.

[34] Bugbee WD, Mizu-uchi H, Patil S, D'Lima D. Accuracy of implant placement utilizing customized patient instrumentation in total knee arthroplasty. Adv Orthop 2013;2013:e891210. https://doi.org/10.1155/2013/891210.

[35] Macdessi SJ, Jang B, Harris IA, Wheatley E, Bryant C, Chen DB. A comparison of alignment using patient specific guides, computer navigation and conventional instrumentation in total knee arthroplasty. Knee 2014;21:406-9. https://doi.org/10.1016/j.knee.2013.11.004.

[36] Ng VY, Arnott L, Li J, Hopkins R, Lewis J, Sutphen S, et al. Comparison of custom to standard TKA instrumentation with computed tomography. Knee Surg Sports Traumatol Arthrosc Off J ESSKA 2014;22:1833-42. https://doi.org/10.1007/s00167-013-2632-7.

[37] Moopanar TR, Amaranath JE, Sorial RM. Component position alignment with patient-specific jigs in total knee arthroplasty. ANZ J Surg 2014;84:628-32. https:// doi.org/10.1111/ans.12674.

[38] Heyse TJ, Tibesku CO. Improved tibial component rotation in TKA using patient-specific instrumentation. Arch Orthop Trauma Surg 2015. https://doi.org/10.1007/ s00402-015-2157-2.

[39] Heyse TJ, Tibesku CO. Improved femoral component rotation in TKA using patient-specific instrumentation. Knee 2012. https://doi.org/10.1016/j.knee.2012.10. 009.

[40] Woolson ST, Harris AHS, Wagner DW, Giori NJ. Component alignment during total knee arthroplasty with use of standard or custom instrumentation. A randomized clinical trial using computed tomography for postoperative alignment measurement. J Bone Jt Surg 2014;96:366-72. https://doi.org/10.2106/JBJS.L.01722.

[41] Thienpont E, Bellemans J, Victor J, Becker R. Alignment in total knee arthroplasty, still more questions than answers. Knee Surg Sports Traumatol Arthrosc 2013; 21:2191-3. https://doi.org/10.1007/s00167-013-2622-9.

[42] www.radiologyinfo.org, Accessed date: 28 June 2016. 